\title{
Cetuximab-Nutzen bei Darmkrebs bestätigt
}

Patienten mit metastasiertem kolorektalem Karzinom profitieren von der Therapie mit dem Antikörper Cetuximab. Deutsche Onkologen haben das jetzt mit aktuellen Daten zweier großer Studien bestätigt.

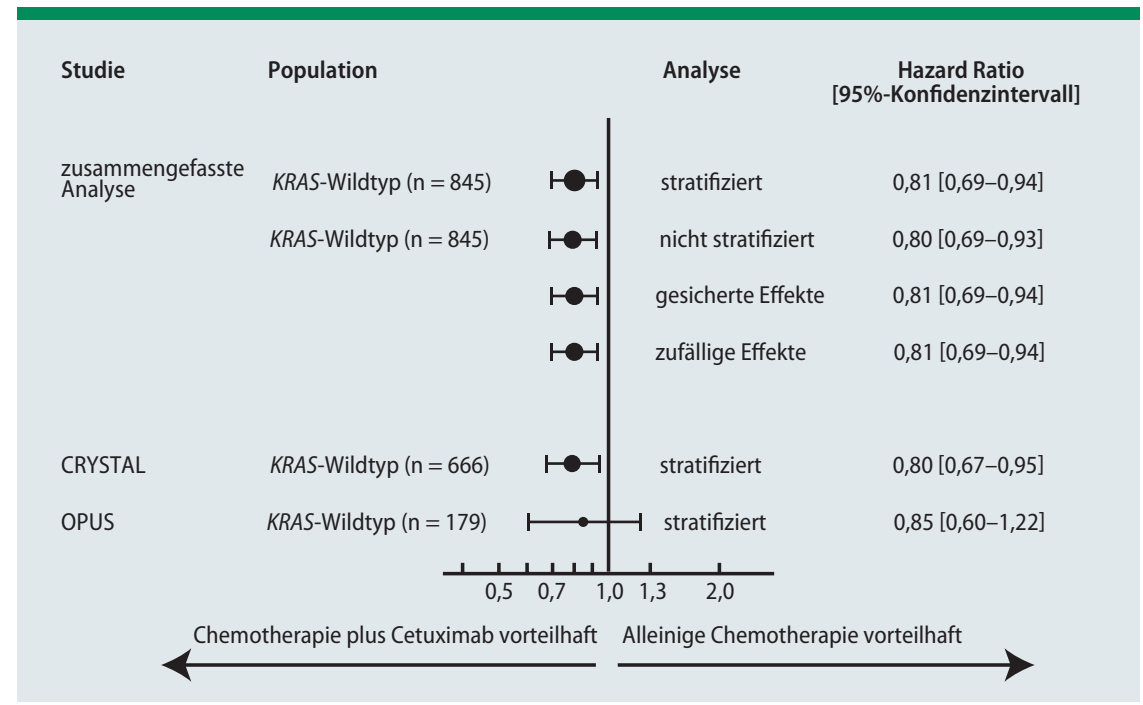

Abb.: Hazard Ratios für das Gesamtüberleben von Patienten mit KRAS-Wildtyp-Tumoren, stratifiziert oder nicht stratifiziert nach dem ECOG-Performance-Status unter Chemotherapie (FOLFIRI/FOLFOX-4) plus/minus Cetuximab.

$\mathrm{D}$ ie beiden randomisierten Studien CRYSTAL und OPUS haben gezeigt, dass sich in der Erstlinientherapie des metastasierten Kolorektalkarzinoms die zusätzliche Applikation des gegen den Rezeptor für epidermalen Wachstumsfaktor (EGFR) gerichteten Antikörpers Cetuximab zu einer Standardchemotherapie günstig auf den Erfolg auswirkt.

Jetzt analysierten Onkologen um Carsten Bokemeyer, Hamburg, aktualisierte gepoolte Daten beider Studien unter dem Aspekt des Gesamt- und des progressionsfreien Überlebens und der Gesamtansprechrate. Insgesamt 845 von 1.535 Patienten beider Studien trugen in ihren Tumoren das KRAS-Wildtyp-Gen, 70 davon außerdem ein mutiertes $B R A F-G e n$.

In der CRYSTAL-Studie enthielt die Chemotherapie außer 5-Fluorouracil (i.v.) und Folinsäure noch Irinotecan (FOLFI$\mathrm{RI}$ ), in der OPUS-Studie dagegen Oxaliplatin (FOLFOX-4).

Im Vergleich zur Chemotherapie allein lag die Wahrscheinlichkeit für ein Ansprechen bei Patienten mit KRAS-WildtypGen, die zusätzlich Cetuximab erhielten, um das 2,16-Fache höher. Mit einer Ha- zard Ratio (HR) von 0,66 wurde das Risiko für eine Progression durch die zusätzliche Antikörpertherapie signifikant um $34 \%$ verringert, das Mortalitätsrisko um $19 \%$ (HR 0,81).

Eine schlechtere Prognose haben dagegen offenbar Patienten mit KRAS-Wildtyp, aber einem mutierten $B R A F-G e n$, und zwar unabhängig davon, ob sie den Antikörper erhalten oder nicht.

Fazit: Die Auswertung der gepoolten Daten zweier großer Studien zur Therapie von Patienten mit metastasiertem Kolorektalkarzinom untermauern die Beobachtung, dass zusätzlich zur Chemotherapie verabreichtes Cetuximab das Gesamtüberleben verbessert und die Ansprechrate erhöht. Von der Kombitherapie profitieren Patienten, deren Tumoren das KRAS-Wildtyp-Gen tragen. Peter Leiner

Bokemeyer $\mathrm{C}$ et al. Addition of cetuximab to chemotherapy as first-line treatment for KRAS wild-type metastatic colorectal cancer: Pooled analysis of the CRYSTAL and OPUS randomised clinical trials. Eur J Cancer. 2012;48(10):1466-75. 